\title{
Los procesos de subjetivación de las nuevas élites culturales. Itinerarios de autonomía e (in)dependencia emocional de mujeres de clase media
}

\author{
Ángel J. Gordo López
}

Universidad Complutense de Madrid. Departamento de Sociología IV

ajgordol@cps.ucm.es

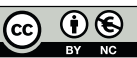

Recibido: 25-10-2012

Aceptado: 10-06-2013

\section{Resumen}

En este artículo se analizan los relatos de vida de dos mujeres de clase media nacidas a finales de los años sesenta en España. En un primer momento se presenta el contexto familiar, a modo de panorámica de la posición social de partida, para ilustrar, seguidamente, la importancia de la educación y de las prácticas de socialización en las trayectorias vitales y afectivas de estas dos informantes, denominadas aquí Alba y Elsa. Este primer momento narrativo, más próximo a las declaraciones de las protagonistas, dará paso a la interpretación de los relatos de vida en un contexto sociológico más amplio. Sus trayectorias vitales y emocionales responden, en parte, a posicionamientos familiares de partida distintos, si bien algunas de sus características comunes se forjan al amparo de un escenario social, con grandes cambios políticos y culturales, en el que coexisten códigos de género, percepciones del mundo y sistemas de valores distintos.

Palabras clave: capital cultural; capital relacional; conciencia de clase; cultura de la excelencia; identidad; madres coraje; participación política; relatos de vida; repertorios de subjetividad; trayectorias afectivas.

Abstract. Subjectification processes of the new cultural elites: Routes of autonomy and emotional in/dependency of middle-class women

In this article we analyze two life accounts of middle-class women born in the late sixties in Spain. We begin by outlining their family background with a view to providing an overall sociodemographic and then illustrate the influence of education and other socialization practices in the emotional and political routes of these two informants to whom we shall refer to as Alba and Elsa. This first narrative moment, which runs closer to the statements and accounts of our protagonists, is followed by the interpretation of their life stories in a broader sociological context. We argue that their emotional and life trajectories partly respond to different starting family positionings. We also suggest that some of their common features are forged under a social scenario which underwent large social changes and where different gender codes, worldviews and value systems coexisted.

Keywords: cultural capital; social capital; class consciousness; excellence culture; identity; mother courage; political participation; life stories; life accounts; subjectivity repertoires; affective trajectories 


\section{Sumario}

$\begin{array}{ll}\text { 1. Introducción } & \text { 3. De la narración a la interpretación } \\ \text { 2. Alba y Elsa } & \text { 4. Conclusiones } \\ & \text { Referencias bibliográficas }\end{array}$

\section{Introducción}

Este trabajo forma parte de un estudio más amplio sobre el papel de las mujeres en la vida social y política de la España contemporánea. El objetivo principal de esta investigación era contribuir a visibilizar la presencia de las mujeres a lo largo del siglo XX y su incidencia en la vida social y política de España a partir del análisis del sentido que confieren las mujeres a sus vidas ${ }^{1}$. Desde una perspectiva atenta a los procesos y a los cambios sociales, proponíamos identificar las líneas de fuerza que configuran problemáticas sociales actuales en las que las relaciones de género y de poder están muy presentes. Para ello utilizamos documentos biográficos, narraciones autobiográficas e historias de vida de mujeres españolas de posición social diferente.

Como señalamos en la introducción de este monográfico (Varela y Gordo, en este volumen), nuestra mirada e interpretación de los materiales analizados están en deuda con los trabajos de Michel Foucault (1979), Norbert Elias (1994), Robert Castel (1999) y, en el panorama sociológico español, con los trabajos genealógicos de Julia Varela y Álvarez-Uría (1997). Nos guiamos por el modo que tienen todos ellos de apelar a la memoria de los conflictos para poder comprender cómo se han gestado las condiciones que conforman el presente y elaborar, así, nuevos conocimientos que puedan ser útiles para explicar lo que está sucediendo en la actualidad. Al igual que el resto de los artículos publicados en este volumen, a la hora de estructurar nuestro análisis, tuvimos en cuenta las dimensiones clásicas de socialización (la familia, la escuela, el trabajo, la pareja y la participación política), inspirándonos, para ello, en los trabajos sobre el posicionamiento y la estructuración social de Pierre Bourdieu (1988, 1997), así como en los estudios de sociología histórica acerca de las cambiantes relaciones entre los sexos de Norbert Elias (1994). Esta perspectiva teórico-metodológica invita a construir una mirada atenta a las tendencias y a los procesos, así como a las discontinuidades, con lo cual se evita reproducir lógicas de progreso lineal o evolutivo. También permite enteder cómo, en una misma época y en una misma sociedad, los mismos procesos pueden afectar de forma distinta a las mujeres de las distintas clases sociales e incluso a las mujeres procedentes de la misma clase social, como veremos en este artículo, en función de sus «capitales» de partida y sus procesos de socialización.

1. Mujeres, cambio social y modos de subjetivación: Análisis sociológico de historias de vida de mujeres en la España contemporánea, Ministerio de Educación y Ciencia, I+D, convocatoria 2004-2007 (Ref. SEJ2006-04140), dirigida por Julia Varela. 
Antes de dar paso a la presentación y al análisis de los relatos de vida, debemos señalar que nuestra práctica metodológica también está en deuda con los trabajos fundacionales que inauguraron la investigación sociológica basada en historias de vida. Entre estos, queremos destacar los trabajos de Ferraroti (1993), Lewis (1969) y Shaw (1996), los estudios igualmente clásicos de Bertaux (1981) y los de Thompson (1978) sobre la historia oral, al igual que el ensayo metodológico de Atkinson (1998) sobre las prácticas de entrevista en la producción y el análisis de historias de vida. También han servido de guía metodológica los análisis de itinerarios vitales de exclusión social y pobreza realizados por Arribas y Serrano (1998), así como el capítulo metodológico de Varela (2008), basado en el trabajo de campo recogido en $A$ Ulfe (2004), sobre historias de vida de varones y mujeres del mundo rural. Igualmente útiles han sido los trabajos de Stanley (1992) sobre la teoría y la práctica de análisis autobiográficos feministas.

\section{Alba y Elsa}

La periodización establecida en la investigación en la que se enmarca el presente trabajo incluía las siguientes etapas: del 98 a la Segunda República, de la República al final de la Guerra Civil, el franquismo y de la transición democrática hasta la actualidad. Nuestra contribución a este estudio se sitúa en la última etapa, la que va desde la transición democrática, tras la muerte de Franco, hasta la actualidad. El análisis de esta etapa comprendía el estudio de mujeres procedentes de clases populares con movilidad social ascendente (véanse Parra y Biglia en este monográfico) y mujeres de clase media, todas ellas nacidas a finales de los años sesenta. No obstante, en este texto, nos limitamos al estudio de dos historias de vida representativas de las mujeres de clase media de este periodo: una primera, basada en el relato de vida de Alba, en la que predominan concepciones de género y relaciones afectivas altamente codificadas, próximas a nociones de amor romántico, idealizaciones de la familia y del mundo emocional individual; y una segunda, la de Elsa, que ejemplifica la importancia de las redes sociales y de los repertorios amplios de socialización a la hora de transitar, en lugar de interiorizar mandatos culturales relacionados con dinámicas de género.

En un primer momento presentamos un resumen del contexto familiar, para ilustrar, seguidamente, la importancia de la educación y de las prácticas de socialización en las trayectorias vitales y afectivas de estas dos mujeres. Esta primera fase, más circunscrita a las declaraciones de nuestras informantes, dará paso a la interpretación de los relatos de vida en un contexto sociológico más amplio, con el propósito de comprender mejor sus posicionamientos diferenciados ante los procesos que, en un escenario sociocultural y político específico, favorecen o dificultan un mayor equilibrio de poder entre hombres y mujeres (Elias, 1994; Foucault, 1985, 1990; Hollway, 1996; Subirats, 2013; Varela, 2011) o posicionamientos más (des)iguales en el sistema cultural formado por el sexo o el género (Rubin, 1975). 


\subsection{Alba: hija de la transición}

Alba procede de una familia de clase media baja y es artista de profesión. Nació a finales de los sesenta en Barcelona, separada y sin hijos. Su madre, con estudios básicos, pero muy inquieta y autodidacta, trabajó como administrativa en un laboratorio hasta que contrajo matrimonio y se dedicó a la educación de Alba y de su hermana, dos años menor que ella. El padre, aparejador en una empresa de construcción, se ausentaba durante largos periodos "para prosperar en el trabajo». Para evitar desplazarse de un sitio a otro con las niñas, la madre decidió mantener el domicilio en Barcelona, una decisión que Alba identifica como una de las muchas renuncias y sacrificios en la vida de su madre, a quien le está muy agradecida, porque fue quien le dijo: “QQuieres estudiar música? ¿Quieres ir al conservatorio?”». Durante los últimos años la relación con su padre se limita a "llamadas en mis cumpleaños, navidades y esas cosas».

Los abuelos maternos eran de Burgos y, según Alba, "para la época, era gente con un nivel». La abuela fue ama de casa y el abuelo, guardia civil, "pro régimen, muy significado». Por el contrario, los abuelos maternos, que se conocieron en Barcelona en la posguerra, tenían una procedencia más humilde: la abuela era aragonesa y señora de la limpieza en épocas de escasez y el abuelo había nacido en un pueblo de Málaga, en el seno de una familia numerosa de agricultores. Resalta que su abuelo "aprendió a leer solo" y militó en la Casa del Pueblo hasta que tuvo que salir huyendo a Barcelona tras un enfrentamiento con los terratenientes del lugar. Alba afirma haberse sentido muy querida por sus abuelos paternos y maternos, si bien destaca la importancia de estos últimos, ya que, según ella, «el núcleo familiar que yo nunca he tenido en mi casa, sí lo tenía con mis abuelos».

\subsubsection{Educación, socialización y vocación profesional temprana}

En la casa de sus padres disponía de una habitación propia llena de libros y juguetes. De aquellos años de infancia recalca la necesidad vital de inventar y crear canciones y letras, así como su facilidad para alegrar la vida a los demás, porque siempre, "cuando llegaba a cualquier lado, era la que cantaba, la que bailaba». A diferencia de otros niños, le encantaba diseñar sus propios recortables y afirma que con dos años tenía «dibujos que son alucinantes [...] y a partir de los seis años hacía cuentos que ilustraba yo, y que luego ponía letras "para mayores de diez años”». Al contrario que a la mayoría de los niños, le gustaba ir al colegio y "estar haciendo cosas», hasta el punto de pedir a los maestros que le mandaran deberes para el verano. Cursó estudios de primaria y bachillerato en un colegio concertado de monjas, y superó los cursos con brillantez, a pesar de las cargas añadidas que suponían las clases extraescolares de inglés en el Instituto Británico, desde los tres años, y de solfeo, piano y canto en el Conservatorio, desde los nueve. No resulta extraño, pues, que, ante nuestra pregunta sobre sus principales recuerdos de infancia, responda que se ve a sí misma yendo de camino al colegio, sin apenas tiempo para jugar, porque, según Alba, cuando «no bajas a la calle, no conoces los juegos, no has 
practicado los juegos, con lo cual llegaba la hora del recreo y tampoco me dejaban jugar demasiado».

Sus compañeras de colegio la consideraban un poco repelente, porque se le daba todo bien, desde las matemáticas y el canto, hasta el teatro y la gimnasia. Dice que su intensiva dedicación a las tareas escolares no estaba movida por el afán de destacar, sino porque, para ella, «lo normal era hacerlo bien». Añade con pesadumbre que nunca tuvo «el grupito ese de dos, tres amigas, que van siempre a todos lados», si bien, con doce años, formó parte de una pandilla en su barrio, algo que recuerda como una de las experiencias más gratas de aquella época. Estaba muy ilusionada por sentirse parte de un grupo, pero los cabecillas de la pandilla no tardaron en excluirla, según Alba, «por mi forma de ser, siempre dispuesta a agradar a los demás». Aunque para ella fue un trauma, "ya tenía claro que yo era quien era, y que estaba por encima de un colectivo». Afirma que no volvería a experimentar «esa sensación de pertenecer a un grupo social».

A este sentimiento de desarraigo e incomprensión, se suma la mala relación con su hermana, para quien ella era «el objeto de su frustración, porque desde pequeña yo era la mona, la inteligente, no sé qué, la cariñosa». En su familia, también se siente una «extraterrestre». La relación tan estrecha con su madre se trunca cuando empieza a salir con un chico, su futuro marido, a la edad de dieciséis años, pues «la tragedia que ella estaba viviendo, toda esa frustración, la canalizó contra mí». Esta situación provoca que Alba, desde muy joven, se refugie en su cuarto y en la música, «porque no podía relacionarme con ellas, porque mi madre y mi hermana hacían piña, y entonces la historia era: “ $¡ E r e s$ igual que tu padre...!"».

La experiencia de soledad en el interior de la familia se vio reforzada por la ausencia de un grupo de iguales. Sin embargo, en momentos posteriores, ya no se siente una extraterrestre, aunque predomina la sensación de soledad, de no sentirse acompañada ni identificada con sus amigos, porque «no hablas el mismo lenguaje que ellos, de modo que tus sentimientos, en el fondo, tampoco pueden ser comprendidos por ellos». Aún así, afirma que nunca ha estado del todo «sola y abandonada», porque «soy una persona tremendamente social y, en el fondo, $[\ldots]$ mi hábitat es el contacto con el otro».

A los nueve años, comienza sus estudios en el Conservatorio, donde se siente ella misma. Afirma que allí «era como más dicharachera para todo», y a los once era capaz de procesar y descifrar el lenguaje musical con facilidad. Con dieciséis años ganó un concurso nacional de jóvenes promesas de la canción y le ofrecieron contratos de trabajo que su madre declinó: «Si quería ser algo, lo que tenía que hacer era continuar mis estudios $y$, cuando realmente estuviese preparada al cien por cien, aspirar a lo mejor».

La falta de relaciones sociales sólidas en su entorno más próximo, en el colegio y en el barrio, junto con las fricciones y el distanciamiento con su madre y su hermana cuando empieza a salir con el primer chico, generan en ella un sentimiento sostenido de desasosiego y profunda soledad, más aún en una persona como Alba, quien se define como «tremendamente social». Este sentimiento de abandono se compensa en parte con percepciones y sen- 
timientos extraños que dice tener desde jovencita, cuando juega «a descubrir qué cartas estaban boca abajo, a mover cosas». Más tarde, experimenta sueños premonitorios y experiencias extrañas, "porque de repente tocabas a alguien y tenías sensaciones de ese alguien o visiones». Señala que hubo gente que la buscó en determinados momentos y que le decían a su madre: «Oye, tu hija es una iniciada». Es esta una parte de sí misma que ha querido dejar al margen, "porque desequilibra mucho, te hacen sentir más rara de lo que eres [riendo]». En momentos posteriores del relato establece un paralelismo entre las tareas de composición musical y sus poderes premonitorios cuando afirma que «a veces, estaba componiendo algo y sabía que estaba bien, que iba a funcionar y tal. Notaba la misma sensación que cuando tenía un sueño premonitorio y, de repente, sin saber por qué, sabía que eso iba a ser realidad».

La necesidad imperiosa de hacer cosas, de crear e inventar, junto con una infancia y una juventud "totalmente copada» por las actividades extraescolares, la obligaron a llevar un ritmo frenético que se ha convertido en una especie de habitus, pues se prolonga hasta el momento actual, porque, según ella: «Me siento feliz haciendo eso. Me siento realizada». Durante los últimos años ha compaginado numerosos conciertos con trabajos de composición y la gestión de su propia empresa y su casa discográfica, además de cargos de representación en distintas sociedades y gremios de las artes escénicas y de la interpretación. A lo largo de su relato subraya su carácter extrovertido y la tendencia a procurar la diversión y el bienestar de su gente, lo cual, según Alba, obedece «a un exceso de necesidad de amor de los demás».

\subsubsection{Vida afectiva y reconocimiento:}

las relaciones de pareja y la falta de referentes familiares

A los once años, recuerda que veranearon con unos amigos de sus padres en la Rioja, en donde se sintió muy cuidada y reconocida, además de tener mucho éxito con los niños, debido, según ella, a ser «la catalana, y es que era muy mona», lo cual le resultó curioso, porque en su barrio se sentía muy "chiquinina», muy frustrada, ya que, a diferencia de las otras niñas, "ya mujeronas», no desarrolló hasta los dieciséis años. Nos confiesa: «estaba muy preocupada, porque» a los niños de su barrio "les gustaban las que tenían tetas». Afirma que siempre ha sido "muy de fijarme en los chicos», sobre todo en el Conservatorio, donde tenía muchos amigos y donde conoció a su primer novio y futuro marido. Según Alba, «nada más verle, supe que iba a ser un hombre muy importante en mi vida». Se casaron y mantuvieron una relación de diez años. Comenta que, por aquel entonces, ella tenía una intensa vida social, porque «me encanta compartir las cosas, la gente», sin embargo, a su ex marido, no le interesaban los eventos sociales, era de su "grupito de amigos».

A lo largo del relato recalca que la ausencia de una familia nuclear ha supuesto para ella una desestabilización emocional importante, sobre todo por la falta de "referencias con respecto a la pareja». También afirma que en las relaciones siempre ha sido la que ha tomado la iniciativa, quien ha "promovido las relaciones con los otros", lo cual le hubiese gustado que fuera diferente, porque 
«si tú eres la que da ese paso, el otro ya nunca te busca». En las relaciones de pareja, se define como "extremadamente impulsiva», como una «locomotora", y comenta que cuando deja de ser «la que tira adelante, la que se inventa», se pierde a sí misma. Así lo ilustra cuando alude a la relación que mantuvo, después de romper con su marido, con una persona que consideró «el hombre de mi vida». Al igual que en su relación anterior, también experimentó un flechazo: «No le había visto, le tenía a mi espalda, y se me hizo un rollo aquí, y me di la vuelta y le vi». No obstante, siente que él no se involucra, lo que, a la postre, le supondrá vivir la relación como «absolutamente destructiva. Estuve a punto de perder mi vida, precisamente porque me quedé en una vía muerta, y no era capaz de moverme a ningún sitio».

Desde hace un par de años Alba convive con una nueva pareja, con una persona muy creativa y dinámica, que le comenta que vivir con ella es muy divertido, porque, según ella, «yo me invento la vida, cada día me invento una cosa, todo me emociona... Pero, es que esa soy yo, ¿`sabes? Me despierto por la mañana contenta, y me encanta hacer felices a los demás. A veces, tengo un poco la sensación de ser adicta, de "¿qué es lo que te hace feliz? No te preocupes, que yo te lo consigo"”.

\subsection{Elsa: nieta de la República}

Elsa, hija única, periodista de profesión y madre de una niña cuya custodia comparte con su ex compañero, nació a finales de los sesenta en la ciudad de Madrid, en una familila de clase media. Hasta el matrimonio, la madre de Elsa tenía un buen trabajo de gestión y administración, antes de obtener por concurso una plaza de funcionaria en un ministerio. Cuando nació Elsa, la madre decidió dedicarse al cuidado de su hija y, más tarde, a trabajos esporádicos. El abuelo materno es oriundo de un pueblo de Extremadura y la abuela, del barrio madrileño de La Latina. Ambos son de procedencia muy humilde, republicanos y socialistas de origen, aunque «no muy significados». El abuelo obtuvo un trabajo bien remunerado en unos prestigiosos almacenes de Madrid, lo que les permitió costear una buena educación privada a la madre de Elsa.

El padre trabajaba como redactor en un periódico y procedía «de una zona muy deprimida» del País Vasco. El abuelo paterno era electricista, "carlista y católico, apostólico y romano». La abuela, ama de casa. Tras unos años en el seminario, el padre abandonó la formación religiosa y se fue a estudiar a Madrid, donde, posteriormente, ejerció la profesión de periodista hasta llegar a ser el redactor jefe de un prestigioso periódico. Los padres de Elsa se conocieron en un acto político en los años cincuenta. Elsa recalca el hecho de que su madre, a pesar de ser «una niña bien que, de pronto, va a un colegio bilingüe», ya militaba en el PCE antes de conocer a su padre: «Iba a Francia y pasaba el Mundo Obrero, y cosas así, y como iba bien vestida y era una niña pija, pues en las aduanas nunca la paraban». En esta misma línea, señala que "es mi madre la que socializa a mi padre en el ámbito político e intelectual de Madrid. Mi padre es un chico de pueblo, cultivado, pero de pueblo». 
En la época del desarrollismo, en los tiempos de los gobiernos tecnocráticos de López Rodó, Ullastres y López Bravo, en la época de las bases americanas, cuando Semprún y Claudín estaban a punto de ser expulsados del Partido Comunista por desviacionistas, el padre entró en una crisis ideológica y propuso iniciar una nueva vida en una comunidad rural en el sur de España. La madre, que no quería trabajar para el aparato del Estado, «está en la misma onda que mi padre y deciden dejarlo todo", a pesar de que, según Elsa, sus padres, al igual que los abuelos maternos, «nunca hayan sido militantes de primera fila». La madre pidió una excedencia en el Ministerio $y$, durante cuatro años, vivieron en esta comunidad. Elsa afirma que entre la progresía que constituía el círculo de amistades de sus padres y, en particular, en aquella comunidad, la fidelidad sexual, en tanto propiedad privada, se vivía con sospecha ideológica. Y añade que su padre se sentía más cómodo en este ambiente, si bien a su madre le «suponía bastantes quebraderos de cabeza».

Regresan a Madrid una vez agotados los ahorros familiares procedentes de un piso en propiedad de la madre. Elsa, siguiendo la trayectoria educativa de su madre, inicia una nueva etapa educativa en un colegio bilingüe. A finales de los setenta el padre retoma el trabajo de redactor y la madre, que ha perdido la plaza en el Ministerio, retoma su antiguo trabajo como secretaria. Con la llegada al poder del PSOE en el ochenta y dos, el padre acepta un cargo político en la Administración y, con ello, retos contrarios a sus planteamientos ideológicos anteriores, los cuales, recuerda Elsa, supusieron un fuerte sacrificio económico y profesional para su madre, a quien «la echan del curro, y como no tiene pensión, ni tiene recursos, pues eso, se convierte en precaria».

Tras un tiempo de convivencia tortuosa, sus padres se separan. Elsa tiene diecisiete años y no vive la separación especialmente mal, pero sí todo el preludio, porque "pasó de ser una familia, un núcleo familiar muy estable, a de repente eso, una madre deprimida que abandona la vida, un padre que está muy a disgusto. Se deteriora el entorno y deja de venir la gente a casa».

\subsubsection{Educación y sociabilidad temprana}

Elsa identifica su contexto familiar de procedencia en la clase media alta. Lleva una vida desahogada, pero austera y consciente del valor de las cosas, porque «desde pequeña, yo sé que las cosas no son gratis, sino que responden a un esfuerzo de trabajo». Es consciente de que la inversión de sus padres «no fue en ladrillos, sino en su educación». Sus años en la comunidad rural los recuerda como una vida asilvestrada, "todo el día en el campo", y en un entorno con otros niños y niñas donde se «debatía muchísimo, se cuestionaba todo de alguna manera». Una vez trascurrida la etapa rural, y de vuelta a Madrid, ingresó, como ya se ha señalado, en un colegio bilingüe en el que convivía con jóvenes "con un nivel adquisitivo muy alto». Las diferencias sociales le resultaban chocantes y trató de sortearlas entablando amistad con los estudiantes más progres, con vidas y niveles adquisitivos similares a los suyos. Como señala Elsa, era como vivir en dos mundos bien distintos, pero no necesariamente 
incompatibles, porque, «a pesar de que iba a un colegio bilingüe, en mi casa no había televisión, no se compraban cosas superfluamente. Pero entre medias me podía ir a pasar el fin de semana a la Moraleja, a un pedazo de casa, con tías sirviéndome con cofia el desayuno. ¡Yo flipaba! Sí, era bastante choque, pero nunca lo llevé especialmente mal».

Desde pequeña el padre le inculca la idea de que, en lugar de buscar la riqueza personal y la paz a través de un hombre o una pareja, tenía que encontrarlas en ella misma, "con tus historias y tal. Lee tus libros y haz tu vida, viaja y conoce gente». En ocasiones, la educación de Elsa en los valores de autonomía e independencia la hacen sentirse como "un bicho raro» frente a sus amigas: «Ellas se maquillaban, yo no. Ellas llevaban tacones, yo no». También recuerda que, al contrario que sus amigas, «tenía muy metido en la cabeza esto de no ser una mujer objeto: los tíos tenían que ser amigos, antes que amantes. Y los tenía que seducir no por mi físico o mis cualidades de mujer, sino por una cosa más intelectual». Este sistema de valores no le permiten creer en el príncipe azul, ni en la idea de «un hombre para toda la vida», ya que, según Elsa, «mi madre también se encargo de "no hagas también lo que yo he hecho, no te supedites a ningún hombre, no lo admires como yo admiro a tu padre y valórate a ti misma por encima de todo"”.

\subsubsection{La universidad, participación política y cultura del esfuerzo}

Durante los años de sus estudios en la universidad participó en la Asamblea de Mujeres y conoció a personas que entendían el papel femenino y las relaciones como ella, «sin haber pasado por la militancia, casi de un modo existencial, pero no educacional». Sin embargo, se alejó de una militancia feminista diciendo que no tardaría mucho en darse «cuenta de que no funcionaba, que veía muchas contradicciones». También participó en el movimiento okupa de finales de los ochenta, cuya idea era tomar edificios deshabitados «y convertirlos en un centro social en el cual el barrio tenga voz, que la gente haga cosas». Ante nuestra pregunta sobre los referentes de este movimiento, afirma que eran grupos de procedencia antifascista, antisistema, muy influidos por el movimiento autónomo alemán, y «sobre todo muy de guerrilla» urbana. Puntualiza que, en estos grupos, las prácticas no eran tan abiertas como era de suponer, ni tan respetuosas con las mujeres. A finales de los ochenta se integró en un grupo de mujeres dentro de este movimiento, con el propósito de cuestionar las dinámicas de género que el propio movimiento reproducía. Elsa apunta que este grupo no fue bien entendido, «más bien poco reconocido». Con la okupación de una casa en Vallecas, el movimiento se abrió a la participación de la clase trabajadora, algunos de ellos maltrechos y marginalizados por la droga, al igual que el ala izquierda del Colectivo de Lesbianas, Gays, Transexuales y Bisexuales de Madrid (COGAM) y antiguos militantes del Movimiento Comunista (MC). Esta apertura permitió la consolidación de dos grandes líneas: la del núcleo duro, más cercana a "la guerrilla del Salvador, lo serio es estar con ETA», y otra línea más experiencial, en la que, como señala Elsa, «el Salvador me queda bastante más lejos que el hecho de que mi amigo salga 
del armario y me cuente sus historias». Años después rompió con la estética femenina dominante en el movimiento y pasó a una postura periférica: «De repente, me maquillo y me pongo tacones y me pongo ropa ajustada y escotes, y encajo fatal en el mundo okupa».

A pesar de la igualdad y del sentido de no propiedad que prevalecen en estos círculos que Elsa sigue frecuentando actualmente, "en el fondo hay bastante conservadurismo en ciertas cosas». Afirma que es habitual que los hombres piensen que "los hijos son cosa de mujeres" y que, entre ellos, haya un hervor generacional permanente: «De hecho, los tíos de mi edad ligan con tías de veintiocho, generalmente, vamos. Las otras, las de mi edad, son compañeras, no son ligues». Entre las mujeres destaca la figura de las madres coraje, valoradas de modo positivo por la comunidad, y a las que Elsa se refiere como mujeres que ostentan el monopolio de la educación y la crianza de los hijos e hijas, porque piensan que "es algo nuestro, somos nosotras y nos obligamos a través de esto, y somos las que realmente decidimos, las que parimos con dolor, las que, en el fondo, sufrimos más». La figura de la madre coraje, procedente "de un núcleo de clase media alta, gente que ha ido a colegios privados», encuentra en la crianza y la educación de los hijos la posibilidad de hacerse valer y, de este modo, contrarrestar el poder de los hombres en su comunidad. Elsa no acaba de entender la manera militante de vivir la maternidad por parte de estas amigas suyas, porque «si yo pienso en un compañero con el que voy a tener un hijo, es que lo máximo que aspiro es a que él asuma las mismas responsabilidades que yo».

En los años de resaca de la movida madrileña, de las grandes decepciones políticas posteriores a la transición (ingreso en la OTAN, huelgas generales, $14 \mathrm{~F}$ ), antesala de años de bonanza económica y la liberación de la vida social y política en el amplio sentido del término, Elsa promovió, con otros amigos, una asociación cultural en un barrio céntrico de Madrid. De repente, el local se convirtió en lugar de encuentro de grupos e idearios hasta entonces incompatibles. En este sentido, enfatiza que lo más bonito de esta iniciativa fue el modo de ayudar a romper la fragmentación y la compartimentación que prevalecía hasta la época entre los distintos grupos activistas de Madrid: «Lo mismo iban marroquíes, que gays, que punkies, que lesbianas, que feministas, o sea, se montó una cosa entre política y a la vez lúdica».

Tras varios años de participación activa en esta iniciativa, Elsa sentía la necesidad de obtener una seguridad económica y vital a través de un trabajo estable, $y$, con veintiocho años, pasó a formar parte de la plantilla de un periódico. Desde entonces, intercala periodos de contratos indefinidos con colaboraciones periodísticas esporádicas.

\subsubsection{Las relaciones de pareja y la maternidad}

A lo largo de su juventud, sale con hombres de distintas esferas y procedencias sociales, desde el guaperas de «ojos azules», el «majete» de los suburbios de Madrid, hasta el hijo de un gran empresario español, «absolutamente desprendido de los afectos» y con el que se siente maltratada. Más tarde se 
enamora de un chaval a quien describe como "super buena persona», que fallece en un accidente de tráfico poco después de iniciar una relación. Tras este fuerte varapalo, vive unos años en los que se siente «muy poco comprometida con la pareja en general». No quiere sufrir y le da miedo centrarlo todo en una sola persona. Más tarde mantiene relaciones abiertas y flexibles en las que dice encontrar cierto equilibrio, "pero vuelvo a acabar deseando a otra gente y dejando la relación [...] por mi necesidad de estar socializando permanentemente».

Tras años de mucho salir, de volcarse al exterior, sin comprometerse con nadie, cuando su vida se aproxima a los cuarenta años, se embarca en una relación de pareja que define como "superconvencional» en la que decide ser madre. Vive la maternidad "de un modo muy esquizofrénico», pues, por una parte, se siente «biológica y mentalmente» inmersa en el cuidado de su criatura $\mathrm{y}$, por otra, las múltiples exigencias en el trabajo, la propia maternidad y una pareja muy absorbente le producen un profundo sentimiento de culpa: siente que no está siendo lo suficientemente generosa, «sobre todo con la niña», que algo le está fallando. Según va creciendo su hija, Elsa experimenta un cierto alivio y recobra en parte su autonomía: «Vuelvo a ser yo, y esta cosa está aquí, ya no me necesita todo el rato, no me demanda, entonces, incluso a veces quiere que no esté [a media risa], incluso yo quiero que ella no quiera que yo esté [a media risa]». Empieza a ser consciente, según ella, de la impostura en la que ha decidido vivir: esforzarse por ser «normal» y vivir en valores socialmente inculcados. Cuando iniciamos el relato de vida Elsa llevaba seis meses separada del padre de su hija, con el que convivió ocho años.

Tras la separación, vuelve a un estilo de vida en el que prima la tranquilidad, tanto en sus nuevas relaciones esporádicas, como en la relación con la hija. Desde esta posición intenta inculcar a su hija el sentimiento de independencia. Quiere estar con ella para disfrutar y comunicarse en lugar de hacerlo desde la dependencia o la culpa: "No quiero que dependa mi vida de ella, tampoco quiero que me necesite más de la cuenta. $\mathrm{Y}$ eso a veces cuesta, porque veo a otras amigas mías que están mucho más volcadas».

Elsa retoma amistades y relaciones sociales del pasado, basadas en la afinidad experiencial, en lugar de identidades y códigos férreos. Así lo expresa cuando se refiere a su amplio círculo de amistades: "A estas alturas de mi vida puedo decir que incluso tengo amigos fachas, que son del Colegio [bilingüe], que son muy conservadores, con los que puedo hablar sin ningún problema, y me gusta hablar con ellos. Hay amigos que tengo de mi entorno [okupación, movimiento autónomo] que jamás hablarían con una persona así». En la parte final de su relato, afirma que, actualmente, entre sus grupos de amigas, además de las madres coraje, también hay «mujeres que son muy feministas, que están en montones de cosas de género, trabajando muchísimo a todos los niveles, y luego algunas que no, que para nada, que el análisis de género incluso no lo entienden. En realidad, me entiendo bien con todas y con todos, porque, a la hora de la verdad, tienes más afinidades que desencuentros ¿no?». 


\section{De la narración a la interpretación}

Alba vive una vida acaparada por el estudio sin apenas tiempo para ser niña. Su madre se vuelca en la educación y la crianza de sus hijas, y sabe detectar y fomentar, a través de la educación extraescolar, las aptitudes artísticas de Alba. El éxito profesional de nuestra informante no aminora un sentimiento de fuerte desequilibrio. Como ella misma afirma:

En lo profesional, soy una persona tremendamente segura y gestiono muy bien las cosas buenas y malas ino? Y pongo todo en su sitio. Sin embargo, en esa parte emocional, pues hay un desequilibrio grande. [...] Entonces, alguien que es así, ¿cómo puñetas se puede llegar en lo personal a ser todo lo contrario? Una persona con una dependencia emocional, cuando yo tengo en mi mano más que muchos hombres y más que otras muchas mujeres, la capacidad para relacionarme con los demás, para poder hacer, para poder crear mi universo, [...], ¿cómo puedo en lo personal, de repente, caer en los tópicos más estúpidos?

Alba considera que «la falta de una familia tradicional» le ha privado de referentes válidos para sus relaciones de pareja, lo que interfiere, a su vez, en la imposibilidad de disfrutar de lleno con sus éxitos profesionales, porque «sé lo que es tener a dos mil personas adorándote y de pie, y aplaudiéndote y demás; después ellos se van con su familia y tú te vas a un hotel y empiezas a llorar en el jacuzzi». La literatura especializada en el análisis del sentimiento de dependencia emocional femenina, de corte psicologista o psicodinámica, remarcaría como una respuesta clásica la tensión que experimenta Alba entre la necesidad de agradar, de complacer, y el deseo de autoafirmación. Nos encontramos, en fin, ante dinámicas que sobrevaloran la familia y la pareja, y que remiten a formas de feminidad basadas en la abnegación, en ponerse al servicio de los otros, así como en la capacidad de entrega, aunque ello suponga la postergación y la renuncia a los deseos y a los proyectos personales (veáse una revisión interesante de estos estudios en Esteban y Távora, 2008).

Al final del relato, Alba señala que ha aprendido a gestionar este desequilibrio a través de la aceptación de su forma distinta de ser y de sentir la vida, "para bien o para mal», de su capacidad ilimitada de sentir y de sufrir. Afirma que ha llegado a comprender que si quería seguir haciendo lo que hacía, que si no quería vivir a medias, "también tenía que aceptar que había esa otra parte terrible que era sufrir también más que los demás, vaya, pero cuando soy feliz también soy mucho más feliz que los demás». Este discurso, próximo a la psicología positiva y la noción de "resiliencia psicológica»" asoma de nuevo cuando

2. Entendida como la capacidad de sobreponerse a contratiempos, al dolor emocional o a traumas y salir fortalecido o fortalecida en el proceso. El concepto de «resiliencia psicológica» ha cobrado un gran protagonismo al amparo de la efervescencia (desde la década de los años noventa) de la emoción (y, por ende, la regulación e instrumentalización de la misma como modelos de apaciguamiento del malestar social generado por dinámicas y estructuras alejadas de lógicas individuales o psicológicas) y el surgimiento coetáneo del fenómeno coaching, la programación neurolingüística (PNL) y las terapias complementarias (psicodrama, conste- 
afirma haber descubierto que «en esencia, soy un ser feliz y energético, y con capacidad de conseguir las cosas que realmente me propongo. Y por eso soy pionera en tantas cosas, posiblemente porque, mientras que hay gente que pone límites a su existencia, yo jamás he tenido la sensación de que existan límites».

Las historias de vida nos brindan la posibilidad de entender los procesos sociales a partir de las explicaciones y las experiencias subjetivas de los informantes, y permiten atisbar otras relaciones y correspondencias biográficas en un contexto social más amplio. Y así, por ejemplo, la sensación acrecentada de soledad y frustración de Alba — una persona que considera el contacto con el otro su principal hábitat- no es ajena a una infancia y a una adolescencia copadas por actividades formativas, ni a una educación tan unilateralmente proyectada al logro personal que le impide socializarse con los niños de su barrio, con el grupo de iguales. La socialización primaria en la familia y las instituciones educativas reforzó su mundo interior, generó incluso las sensaciones paranormales que, en última instancia, revierten en nociones de un yo fuerte, una especie de omnipotencia del yo alimentada por un sentimiento mesiánico, sin límites, que deriva tanto en sentimientos positivos y negativos como en la realización artística. Estos procesos de subjetivación quedaron reforzados a lo largo de las incursiones frustradas de Alba en grupos de pertenencia y afinidad, tanto en las compañeras de colegio (por ser empollona y hacerlo todo bien), como en la pandilla del barrio ("por mi forma de ser, siempre dispuesta a agradar a los demás»). Estas carencias en la sociabilidad primaria le impidieron «experimentar esa sensación de pertenecer a un grupo social». Estos procesos son relevantes a la hora de entender cómo se fraguó su posterior sensación de ser diferente y, por ende, su afianzamiento en una toma de conciencia respecto a su forma de ser diferente. Así lo expresa Alba cuando, tras ser excluida de la pandilla del barrio, dice darse cuenta de que «yo era quien era, y por eso estaba por encima de un colectivo». El afianzamiento de estas relaciones sociales, $y$ su forma de alimentar una forma de ser «diferente», se agranda aún más con la ruptura de la relación fusional con su madre en el momento en el que Alba empieza a salir con su primer novio. La madre vive este espacio afectivo y de autonomía de su hija como una amenaza y una traición, porque siente en aquel momento, según nuestra protagonista, que todos sus esfuerzos y sus sacrificios vitales han sido invertidos en el cuidado y en la educación de sus hijas, especialmente de Alba, a la que considera su obra de arte, además de un asidero, o refugio vital, ante un matrimonio cuya ruptura era encubierta por unos tiempos de democracia incipiente, con códigos de género muy codificados y desiguales, más aún entre las clases medias emergentes, con mayor capital económico que cultural. A partir de este momento la madre identifica a Alba con la figura ausente («eres igual que tu padre»), causa primigenia de todo el desequilibrio familiar y personal (o «fantasma»), lo cual la sitúa en una posición de enfrentamiento ante la díada formada por su madre y su hermana menor.

laciones familiares, reiki, etc.), al igual que la psicología positiva y transpersonal (para una visión crítica de estas nuevas tendencias, véase Parker, 2010, y Álvarez-Uría et al., 2010). 
Como señala Alba, en ese momento, la tragedia personal que estaba viviendo la madre, «toda esa frustración, la canalizó contra mí». No de extrañar, pues, que la niña se refugie en su cuarto, en sus estudios de música, en su mundo de percepciones extrañas «porque no podía relacionarme con ellas, porque mi madre y mi hermana hacían piña».

Elsa, al igual que Alba, procede de un entorno familiar de clase media, pero parte de una posición más elevada en la estratificación social. Elsa, a diferencia de Alba, posee un mayor capital cultural y relacional que económico, lo que le proporciona una amplia red de amistades que seguirá ampliando a lo largo de su educación y posterior participación política (véase el análisis realizado por Pilar Parra en este mismo número monográfico para una presentación más detallada de los distintos tipos de capitales según Bourdieu, 1997). Este posicionamiento social de partida, sumado a una educación familiar que le inculca valores de autonomía e independencia, le permiten adoptar una distancia crítica hacia las relaciones de género y hacia ideologías fuertemente codificadas. También la predisponen a apreciar la realidad desde múltiples lecturas, lo cual da paso a lógicas y relaciones más abiertas, mediadas por una revalorización del mundo experiencial y la empatía. Asume su identidad social desde discursos y prácticas marcados por la lógica de la afinidad, en lugar de la búsqueda de un yo interior fuerte (como en el caso de Alba). No obstante, la diversidad de repertorios de Elsa y su socialización primaria en una familia socializada en la cultura del esfuerzo, a diferencia de muchos de sus amigos y amigas, le permiten transitar a través de los estilos de pensar y de vivir asociados al paradigma de la precariedad desde finales de los años ochenta y compaginar el trabajo remunerado, la diversión y la movilización política ${ }^{3}$.

Lejos de marginarla o restarle oportunidades, este tipo de actitud durante la transición española le permite ampliar y diversificar aún más sus afinidades y sus redes (capital relacional), a la par que sus recursos y sus repertorios de subjetividad (entendidos como los recursos disponibles para pensar y actuar sobre uno mismo y sobre las demás personas — Rose, 1990). Así lo apreciamos en el relato de Elsa cuando afirma saberse distinta al resto de las niñas en su adolescencia, lo cual no evita que ella también se enganche al guaperas de turno o, más tarde, establezca una relación «superconvencional» con el padre de su hija. Este constante transitar, en lugar de interiorizar mandatos culturales respecto a las relaciones de género, la pareja y, entre otros, la maternidad, queda claramente manifiesto en la siguiente declaración de Elsa cuando afirma que «el mundo es más variado que eso; hay muchas lecturas dentro de un orden».

3. En otro lugar y momento previo a la gran crisis financiera, en el intento de abordar fenómenos relacionados con la psicologización de la cultura, utilizamos el concepto «ensimismados» para intentar comprender la adscripción de algunos sectores de jóvenes, principalmente de clases medias, a estilos de pensar y vivir próximos a la noción de "precariedad» que se forja en décadas pasadas (Álvarez-Uría et al., 2008, 2010). Para la realización de este trabajo, encontramos un excelente referente en los trabajos de antropología cultural de Douglas (1998) y su noción de outsiders. 
Asimismo, queda patente en sus participaciones políticas (asamblea de mujeres, movimiento de okupación), a las que ella misma alude a lo largo de su relato con los calificativos de "periférica», "satélite» o "no significada» (al igual que el posicionamiento político de sus padres y de sus abuelos maternos), porque, según Elsa, «me resulta imposible adscribirme a un solo grupo». Por tanto, los múltiples repertorios de subjetividad le proporcionan recursos para contrarrestar lógicas muy codificadas, bien sean de participación política o estilos de pensar y vivir las relaciones de género, de pareja o de maternidad.

En lo relativo a los procesos de transitar (Elsa) o adscribirse y gestionar dilemáticamente a los mandatos culturales o códigos de género estereotipados (Alba), Cristina Santamarina (2004) recuerda que, a pesar de los muchos logros conseguidos a favor de relaciones de género más simétricas, las mujeres de clases medias y medias altas encuentran un obstáculo común, la maternidad, hasta la fecha insalvable para la mayoría y que las devuelve a una noción de «feminidad verdadera». De manera similar Elisabeth Badinter (2011) plantea que el tan venerado «instinto maternal» ha adoptado un cariz exacerbado en el mundo occidental industrializado, incluso dentro de sectores aparentemente progresistas. La defensa a ultranza de la maternidad, envuelta ahora en discursos ecofeministas que celebran procedimientos naturales en el parto, el pecho a discreción y los pañales reciclables suponen, según Badinter (2011), un retroceso y una vuelta a valores tradicionales, una regresión a formas «naturales» de maternidad, en la tendencia cada vez más patente de hacer del hijo o de la hija «su pieza de arte», máximo exponente de la autorrealización maternal y, por ende, de «la nueva mujer». Una tendencia que, según los análisis de Badinter, debe ser entendida como una respuesta ante los sinsabores de la política laboral actual, más exacerbada desde la gran crisis financiera, y también en respuesta a las posiciones actuales maltrechas de algunas de las madres, mujeres militantes y mujeres combativas (como la madre de Elsa), quienes, bajo el discurso de la libertad sexual tan frecuente en determinados entornos progresistas en décadas pasadas (Hollway, 1996), ven cómo las diferencias y las asimetrías quedan subsumidas en discursos más amplios de transformación social (como hemos apreciado en el caso de la madre de Elsa y su experiencia en la comunidad rural o la incursión de Elsa en el movimiento de okupación) y que, en última instancia, reproducen fuertes asimetrías de género (por ejemplo, la posición actual de madres coraje entre estos colectivos del movimiento autónomo y de okupación). Por su parte, en los márgenes más tradicionales con menor capital sociocultural, como sucede en el entorno familiar de Alba, prevalecen códigos de género más codificados y explícitos, acompañados en épocas pasadas de la figura de la madre coraje (a la vieja usanza), abnegada y volcada al cuidado y a la educación de sus hijas. En dichos entornos apreciamos como el yugo de «la normalidad», sus códigos morales y de género dominantes, permitieron que mujeres con gran inquietud, como la madre de Alba, se vieran abocadas estructuralmente a la posición de madres coraje de la transición (casadas pero solteras, solas pero dependientes), sin poder desarrollar sus inquietudes ni habilidades hasta bien avanzadas sus vidas. 
Estas dinámicas estructurales también afectan al modo en que Alba, tan segura y resolutiva en lo profesional, y tan dependiente e inestable en las relaciones de pareja, encuentra la respuesta última de su forma de ser en la falta de referentes familiares propiciada por un padre ausente, en lugar de, por una parte, en su adscripción y reproducción de códigos de género altamente codificados $y$, por otra, en un espacio de relación fusional con la madre, quien, al igual que las nuevas madres de las que nos habla Badinter, también hizo de Alba su propia obra de arte, su asidero vital ante un matrimonio cuya ruptura era encubierta por unos tiempos de democracia incipiente, con reglas de género muy codificadas y desiguales, más aún entre las clases medias emergentes con mayor capital económico que cultural. Algo parecido ocurre en la biografía de Elsa y la insistencia de su madre de no depender de un solo hombre, como hizo ella con su padre, lo que, en última instancia, le supuso abandonar su profesión, su proyecto vital, para terminar, tras la separación del padre de Elsa, en una decrepitud vital y anímica.

Las figuras de la madre coraje, bien sea en la modalidad de la «nueva mujer» (o madre intensiva) envuelta en valores ecofeministas, y en respuesta a la crisis económica y desafección política (Badinter, 2011) o como resultado de luchas explícitas de género (en los colectivos procedentes del movimiento autónomo y de okupación de Madrid), coexisten en el escenario actual, a pesar de su muy distinta procedencia, lo cual, a nuestro parecer, debería ser motivo de un análisis sociológico más detallado.

\section{Conclusiones}

A partir de sus relatos biográficos hemos "construido» las historias de vida de dos mujeres de clases medias con perfiles socioeconómicos similares pero con marcadas diferencias en lo relativo a la formación cultural, las redes sociales y los niveles de participación sociopolítica. En lo relativo al contexto familiar, Alba procede de un hogar con escasa vida social fuera del entorno familiar, con marcadas diferencias en el posicionamiento social de los padres: una madre de familia humilde, con estudios básicos, dedicada a sus labores, y un padre con estudios técnicos y profesión cualificada procedente de una familia de clase media baja conservadora. Por el contrario, en la familia de Elsa prevalece el capital sociocultural (inversión en cultura, intensa vida sociopolítica, amplias y diversas redes de amistades) sobre el capital económico y existen diferencias menos marcadas entre el capital cultural de partida del padre (estudios superiores) y el de la madre (estudios medios en colegio bilingüe). Por ello podríamos decir que la familia de Alba es representativa de la clase "media» española tradicional, producto de la transición hacia una democracia incipiente, mientras que la familia de Elsa es más típica de las nuevas clases medias, de la "progresía» española, afín a ciertos valores republicanos que incluyen una apuesta por relaciones de género y niveles educativos más igualitarios y autónomos. De ahí que hayamos titulado nuestra interpretación de sus relatos de vida, respectivamente, con las denominaciones «hija de la transición» (Alba) y «nieta de la República» (Elsa). 
Los posicionamientos familiares de partida afectan inevitablemente a la socialización primaria y secundaria, así como a los estilos de pensar y de vivir el mundo emocional, además de las relaciones de género. Como hemos señalado, la escasa sociabilidad de Alba en su infancia y adolescencia, salvo en la esfera profesional del conservatorio, además de su nula participación en actividades sociales y políticas, encuentra una fuerte correspondencia con su forma de interiorizar y hacer suyos relaciones y códigos estereotipados de género en sus relaciones de pareja y el modo que explica sus vivencias negativas de la misma, en primer lugar, a partir de la falta de referentes familiares (de un padre ausente) y, más tarde, el modo que tiene de resolver la situación dilemática que ella misma establece entre sus grandes dotes y su trayectoria profesional y el malestar y la dependencia emocional que experimenta en la pareja, a través de su capacidad ilimitada de sentir y sufrir, como estrategia de aceptación de su manera "distinta" de ser y sentir la vida, "para bien o para mal», y como parte de un discurso adscrito al plano psicológico. La gestión de este tipo de situaciones dilemáticas, el dominino de las mismas a través de la flexibilización de las situaciones sociales o, en el caso de Alba, a través de la capacidad ilimitada de sentir y sufrir, encuentra correspondencia con los análisis de Erving Goffman (2000) sobre el rubor en la vida social del individuo, cuya función sería mantener la identidad de la persona al enfrentarse a situaciones problemáticas, flexibilizando las estructuras o las situaciones sociales, tras una previa pérdida transitoria del control o de la seneridad.

El relato de vida de Alba permite comprender tendencias sociales actuales. Tras largas décadas de deterioro del tejido y de las afinidades sociales, coincidiendo con la primera gran crisis del Estado del bienestar a principios de la década de los setenta, y con la instauración de las lógicas liberales (años ochenta) y neoliberales (años noventa), el individualismo de siglos pasados, inherente a los procesos de modernización, dio paso a las lógicas del new age en coexistencia con la efervescencia de las emociones, todo ello acelerado por la gran crisis financiera, así como por la psicologización de la cultura y de lo social, por el auge, en fin, de una cultura psicológica (Álvarez-Uría, 2005; Varela, 2006). Coincidiendo con el capitalismo neocon, nuevas versiones de la psicología positiva y de la psicología transpersonal coadyuvan a encerrar nuestras vidas en los laberintos del yo (Parker, 2010) (sobre la psicologización del mundo social, véanse también los trabajos de Rose, 1990, y Rendueles, 2005).

En el caso de Elsa, las redes sociales de partida que le proporcionó el entorno familiar, ampliadas a lo largo de su socialización posterior o secundaria, además del sentido de autonomía e independencia que le inculcaron sus padres, le ayudaron a romper con nociones naturalizadas y naturalizantes de feminidad, en lugar de interiorizarlas y hacerlas suyas. Su fuerte capital relacional y, por ende, sus múltiples repertorios de subjetividad, le permitieron transitar a través de mandatos culturales reavivados durante las últimas décadas, tales como el instinto maternal o la "maternidad intensiva» (Badinter, 2011), en lugar de interiorizarlos o hacer de los mismos un rasgo identitario de "feminidad verdadera» (Foucault, 1985; Santamarina, 2004). También le confieren 
versatilidad a la hora de enfrentarse a distintas relaciones de pareja, formas de activismo, además de permitirle conjugar amistades a menudo incompatibles, y redes sociales y de apoyo emergentes como las redes de mujeres (véase Castells y Subirats, 2008; Subirats, 2013).

Estas diferencias quedan en parte diluidas si apreciamos la ausencia en ambas biografías de un relato del yo coherente y consistente, de un proyecto vital claro (Goffman, 2000; Sennett, 2000; 2003), producto de un escenario compartido en el que se produjeron cambios acelerados, la transición española, y en el que coexistieron diferentes códigos de género, distintas percepciones del mundo, distintos sistemas de valores, en fin, distintos estilos de vida. Ambos relatos, a pesar de sus diferentes perfiles sociológicos, muestran procesos de subjetivación un tanto fragmentarios, muy dependientes de las circunstancias, algo que suele ser frecuente en las nuevas clases medias con expectativas de ascenso. Según los estudios de Pinçon y Pinçon-Charlot (1997, 2000), en el momento actual, son únicamente las clases altas, las elites representativas de la cultura de la excelencia, las que manifiestan una conciencia de clase clara y consciente.

Por último, debemos decir que nuestra interpretación de los relatos de vida de Alba y Elsa se aleja de cierres interpretativos, de lecturas psicológicas como las de Esteban y Távora (2008), y encuentra referentes válidos en los estudios sociológicos recientes sobre las mujeres de clases medias ascendentes de Cristina Santamarína (2004) y Elisabeth Badinter (2011), como hemos señalado. No obstante, consideramos importante reparar en dimensiones sociológicas básicas, sus relaciones con los capitales económicos y socioculturales de partida, y el desarrollo de su posicionamiento social a lo largo de la sociabilidad primaria (familia, escuela) y secundaria (amistades, participación política, instituciones, etc.) (Álvarez-Uría y Varela, 2009; Bourdieu, 1988; Varela, 1998, 2011). Estas dimensiones son especialmente importantes en la construcción y el análisis de las historias de vida, como las presentadas aquí, en la medida en que permiten dilucidar correspondencias entre itinerarios y trayectorias de autonomía e (in) dependencia emocional y determinados procesos de subjetivación ${ }^{4}$.

\section{Referencias bibliográficas}

ÁlVAREZ-URía, Fernando (2005). «Viaje al interior del yo: La psicologización del yo en la sociedad de los individuos». Claves de la Razón Práctica, 153, 61-67.

ÁlVAREZ-URÍA, Fernando y VARELA, Julia (2009). Sociología de las instituciones: Bases sociales y culturales de la conducta. Madrid: Morata.

Álvarez-Uría, Fernando; VArela, Julia; Gordo, Ángel y Parra, Pilar (2008). «El estudiante de psicología: La socialización profesional de los futuros psicólogos y la cultura». Revista de la Asociación Española de Neuropsiquiatría [en línea], 28 (1), 167-196. <http://dx.doi.org/10.4321/S0211-57352008000100011>.

4. Quisiera agradecer a Julia Varela y a Fernando Álvarez-Uría sus comentarios y sus aportaciones a este texto y, en particular, sus puntualizaciones sobre la conciencia de clase de las culturas de excelencia. 
- (2010). «Psychologised life and thought styles». Annual Review of Critical Psychology, 8, 11-28. De Vos, Jan y Gordo LÓPEZ, Ángel Juan (eds.). Monográfico «Psychology under Scrutiny» [en línea]. <http:/www.discourseunit.com/arcp8/arcp8full.pdf>.

Arribas, Ana y SERrano, Amparo (1998). ¿Pobres o excluidos?: El IMI en perspectiva comparada. Madrid: Visor / Argentaria.

ATKInSOn, Robert (1998). The Life Story Interview. Londres: Sage.

BADINTER, Elisabeth (2011). La mujer y la madre: Un libro polémico sobre la maternidad como nueva forma de esclavitud. Madrid: Esfera de los Libros.

BerTaux, Daniel (1981). Biography and Society: The Life History Approach in the Social Sciences. Londres: Sage.

Bourdieu, Pierre (1997). Razones prácticas: Sobre la teoría de la acción. Barcelona: Anagrama.

- (1988). La distinción: Criterio y bases sociales del gusto. Madrid: Taurus.

CASTEL, Robert (1999). Las metamorfosis de la cuestión social. Buenos Aires: Paidós.

CASTELls, Manuel y SuBIRATS, Marina (2008). Mujeres y hombres: ¿Un amor imposible? Madrid: Alianza.

Douglas, Mary (1998). Estilos de pensar: Ensayos críticos sobre el buen gusto. Barcelona: Gedisa.

Elias, Norbert (1994). «El cambiante equilibrio de poder entre los sexos». En: Conocimiento y poder. Madrid: La Piqueta. Edición y traducción de Julia Varela.

Esteban, María Luz y TÁvora, Ana (2008). «El amor romántico y la subordinación social de las mujeres: revisiones y propuestas». Anuario de Psicología, 39 (1), 59-73.

FERRAROTI, Franco (1993). «Las biografías como instrumento analítico». En: MARINAS, José Miguel y SANTAMARINA, Cristina (eds.). La historia oral: métodos y experiencias. Madrid: Debate.

Foucault, Michel (1979). Microfísica del poder. Madrid: La Piqueta. Edición y traducción de Julia Varela y Fernando Álvarez-Uría.

- (1985). El sexo verdadero. Madrid: Revolución. Selección de Antonio Serrano.

- (1990). Tecnologías del yo y otros textos afines. Barcelona: Paidós.

GOFFMAN, Erving (2000). «Rubor y organización social». En: DíAz, Félix (ed. y trad.). Erving Goffman, Harvey Sacks, Aarón Cicourel, Melvin Pollner: Sociologías de la situación. Madrid: La Piqueta.

HollwaY, Wendy (1996). "Hacer el amor sin contracepción: Hacia una teoría para el análisis de las explicaciones». En: GORDO LÓPEZ, Ángel J. y LiNAZA, José Luis (eds.). Psicologías, discursos y poder (PDP). Madrid: Visor.

LEWIS, Oscar (1969). Los hijos de Sánchez. México: Fondo de Cultura Económica.

PARKER, Ian (2010). La psicología como ideología: Contra la disciplina. Madrid: Libros de la Catarata.

PinÇOn, Michel y PInÇOn Charlot, Monique (1997). Voyage en grande bourgeoisie. París: Presses Universitaires de France.

- (2000). Sociologie de la bourgeoisie. París: Éditions La Découverte.

RENDUELES, Guillermo (2005). Egolatría. Oviedo: KRK.

Rose, Nikolas (1990). Governing the Soul: The Shaping of the Private Self. Londres: Routledge.

Rubin, Gayle (1975). "The traffic in women: Notes on the political economy of sex». En: ReITER, Rayna (ed.). Toward and Anthropology of Women. Nueva York: Monthly Review Press.

SANTAMARINA, Cristina (2004). «Conflicto de géneros y construcciones discursivas». En: CIMOP [en línea]. <http://www.cimop.com/articulos/Articulos.html>. 
SENNETT, Richard (2000). La corrosión del carácter: Las consecuencias personales del trabajo en el nuevo capitalismo. Barcelona: Anagrama.

- (2003). El respeto: sobre la dignidad del hombre en un mundo de desigualdad. Barcelona: Anagrama.

SHaw, Clifford R. (1966). The Jack-Roller: A delinquent boy's own story. Chicago: The University of Chicago Press.

STANley, Liz (1992). The Auto/biographical I: The Theory and Practice of Feminist Autobiography. Manchester: Manchester University Press.

SuBIRATS, Marina (2013). Forjar a un hombre, moldear a una mujer. Barcelona: Aresta.

Thompson, Paul (1978). The Voices of the Past: Oral History. Oxford / Londres: Oxford University Press.

VARELA, Julia (1998). El nacimiento de la mujer burguesa. Madrid: La Piqueta.

- (2004). A Ulfe: Socioloxia dunha comunidade rural galega. La Coruña: Sotelo Blanco Edicions.

- (2006). «El descubrimiento del mundo interior». Claves de la Razón Práctica, 161, 42-48.

- (2008). «Historias de vida; la crisis del mundo rural». En: GORDO LÓPEZ, Ángel J. y SERrano Pascual, Araceli (coords.). Estrategias y prácticas cualitativas de investigación social. Madrid: Pearson.

- (2011). Mujeres con voz propia: Carmen Baroja y Nessi, Zenobia Camprubi Aymar y María Teresa León Goyri: Análisis sociológico de la biografía de tres mujeres de la burguesía liberal española. Madrid: Morata.

VArela, Julia y ÁlvareZ-Uría, Fernando (1997). Genealogía y sociología: Materiales para repensar la modernidad. Buenos Aires: El Cielo por Asalto. 